\title{
Wafer-Level Heterogeneous 3D Integration for MEMS and NEMS
}

\author{
Frank Niklaus *, Martin Lapisa, Simon J. Bleiker, \\ Valentin Dubois, Niclas Roxhed, Andreas C. Fischer, \\ Fredrik Forsberg, Göran Stemme \\ Microsystem Technology Lab (MST) \\ KTH Royal Institute of Technology \\ 10044 Stockholm, Sweden \\ *frank.niklaus@ee.kth.se
}

\author{
Daniel Grogg, Michel Despont \\ IBM Research-Zurich, \\ 8803 Rüschlikon, Switzerland \\ dpt@zurich.ibm.com
}

\begin{abstract}
In this paper the state-of-the-art in wafer-level heterogeneous 3D integration technologies for micro-electromechanical systems (MEMS) and nano-electromechanical systems (NEMS) is reviewed. Various examples of commercial and experimental heterogeneous $3 \mathrm{D}$ integration processes for MEMS and NEMS devices are presented and discussed.
\end{abstract}

Keywords-microelectromechanical system, MEMS; wafer-level heterogeneous integration; More-than-Moore; NEMS; nanoelectromechanical system; self-assembly; transfer printing

\section{INTRODUCTION}

This paper reviews the state-of-the-art of wafer-level heterogeneous 3D integration technologies and platforms suitable for MEMS and NEMS devices. Most MEMS and NEMS transducers require electric interfacing to the outside world, which is typically done by electronic integrated circuits (ICs) that are combined with the MEMS or NEMS transducer. Wafer-level heterogeneous 3D integration is defined within this paper as wafer-to-wafer and chip-to-wafer joining, processing and interconnecting materials and components that are prepared with different technologies including MEMS, emerging NEMS, photonics and electronic integrated circuits (ICs) [1]. Thus, heterogeneous 3D integration technologies allow the combination of high-performance MEMS and NEMS materials on top of standard IC wafers for complex systems that are not possible to manufacture with other micromanufacturing techniques.

\section{WAFER-LEVEL HETEROGENEOUS 3D INTEGRATION FOR MEMS AND NEMS}

Heterogeneous 3D integration technologies for MEMS and NEMS can be placed in the two categories: (1) heterogeneous integration using via-first (or anchor-first) processes as exemplified in Fig. 1 and (2) heterogeneous integration using via-last (or anchor-last) processes as exemplified in Fig. 2. In heterogeneous integration using via-first processes, the vias establishing mechanical and electrical contacts between components on the different substrates are defined during the bonding process. On the contrary, in heterogeneous integration using via-last processes the components are first bonded to each other and the vias establishing mechanical and electrical contacts between the components on the different substrates are defined thereafter.

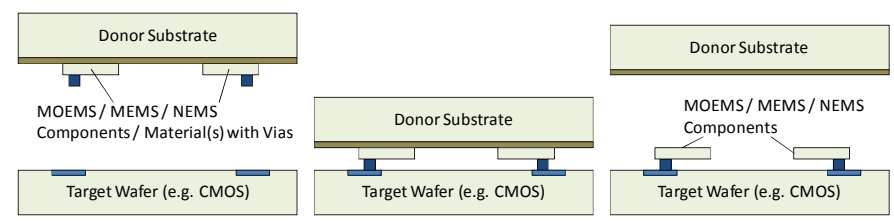

Figure 1. Example of heterogeneous 3D integration using a via-first process.

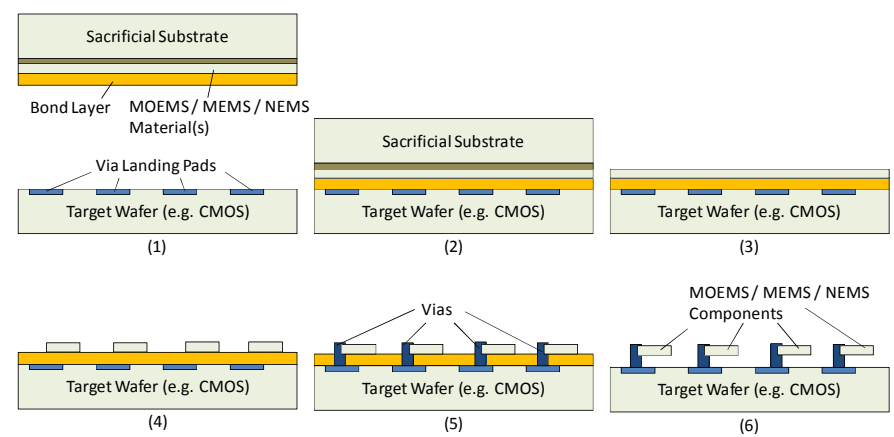

Figure 2. Example of heterogeneous 3D integration using a via-last process

Both, via-first and via-last heterogeneous $3 \mathrm{D}$ integration platforms are being commercially developed. The commercially most developed via-first heterogeneous 3D integration platform is used by InvenSense, Inc (US) for highvolume manufacturing of 1,2 and 3-axis gyroscopes. Fig. 3 shows the InvenSense platform technology in which the mono-crystalline silicon MEMS sensor (in this case a gyroscope) and parts of the MEMS sensor package are prefabricated together on one wafer and subsequently bonded to the CMOS IC wafer that contains an etched cavity as indicated in Fig 3. The bonding and sealing is done directly to the top of the CMOS $\mathrm{Al}$ metal layer using an $\mathrm{Al} / \mathrm{Ge}$ eutectic bonding process $[1,2,3]$. Fig. 4a shows a cross-sectional image and Fig. $4 \mathrm{~b}$ a top view of a commercial gyroscope that has been manufactured with this technology [3]. A commercially available via-last heterogeneous $3 \mathrm{D}$ integration platform technology has been presented by semiconductor foundry TSMC, Ltd (Taiwan) [4]. The process flow for this

This work has been partially funded from the European $7^{\text {th }}$ Framework Programme (EU-FP7) under grant agreements FP7-NEMIAC (no.288670) and by the European Research Council through the ERC Advanced Grant xMEMs (no.267528) and through the ERC Starting Grant M\&M's (no.277879). 
standardized MEMS technology platform is shown in Fig. 5 and can be used to implement IC-integrated MEMS devices such as inertial sensors. Fig. 6 shows evaluation device structures that have been manufactured with the MEMS on IC integration platform.

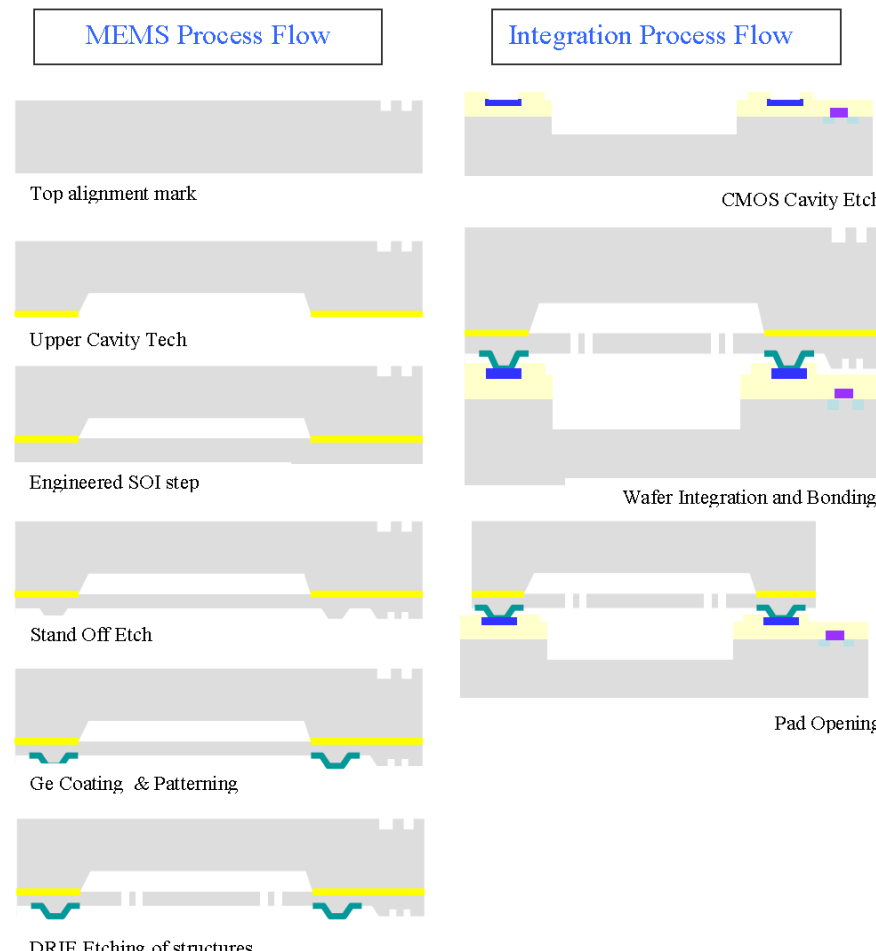

Figure 3. Via-first heterogeneous integration platform (Nasiri-fabrication) from InvenSense which is used for high-volume manufacturing of commercially available gyroscopes. $[1,2]$

Generally, the main advantages of via-first processes are that the components can be completely manufactured prior to bonding and integration and that the integration can essentially be done in a single-step bonding process as depicted in Fig. 3. Disadvantages of via-first processes are that they all require aligned substrate-to-wafer bonding, which adds process complexity and has limitations in the achievable post-bond alignment accuracies [5]. Also, the implementation of reliable via bonding processes for vias with dimensions of below 10 $\mu \mathrm{m}$ seems challenging. In contrast, via-last processes hold promise for MEMS and NEMS with extremely small device dimensions in the nm-range, very small via dimensions in the sub- $\mu \mathrm{m}$-range and placement accuracies of the components on the target wafer in the nm-range. The distance between the device membrane and the substrate surface can be accurately defined in a large interval between $100 \mathrm{~nm}$ and several tenths of $\mu \mathrm{m}$ by the bond layer thickness. In addition, via-last integration schemes that rely on wafer bonding processes that have no precise substrate-to-wafer alignment requirements can be less complex and costly. In the following two sub-sections, a number of via-first and via-last heterogeneous integration platforms are presented that have been described in literature. (a)
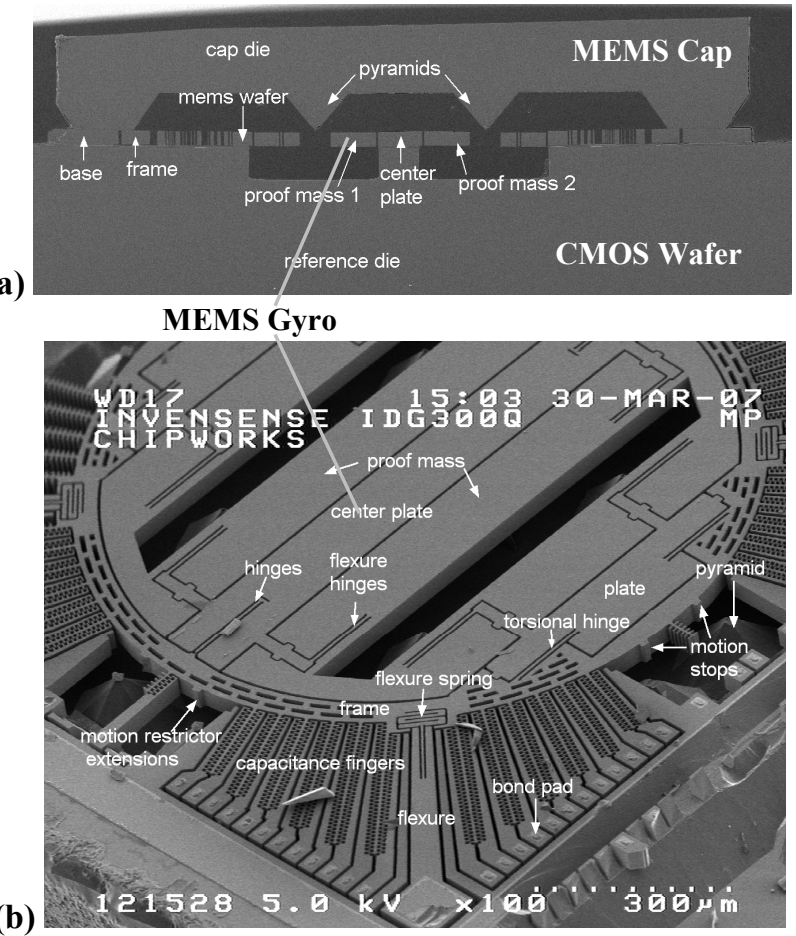

Figure 4. Commercially available gyroscope integrated with CMOS ICs using the via-first heterogeneous integration platform (Nasiri-fabrication) depicted in Fig. 3. [1, 2, 3]
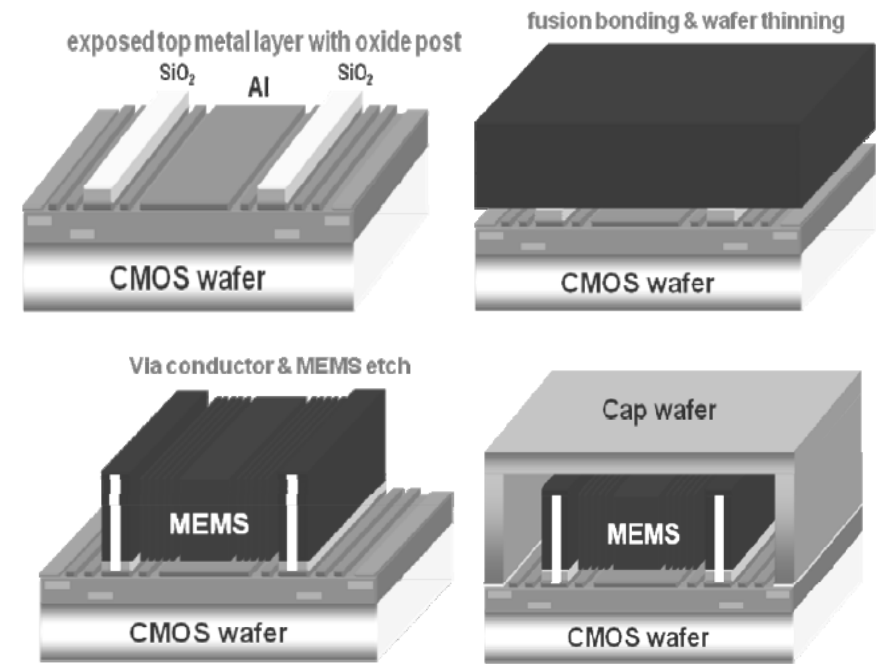

Figure 5. Standardized via-last heterogeneous integration platform from TSMC targeted at inertial sensor applications. [4]
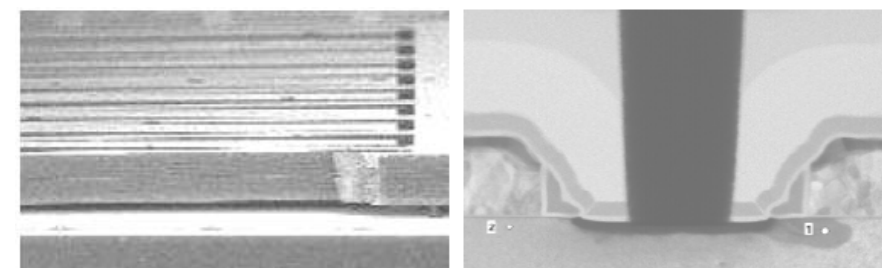

Figure 6. Evaluation test structures from the via-last heterogeneous integration platform shown in Fig. 5 from TSMC. [4] 


\section{A. Wafer-Level Heterogeneous 3D Integration Platforms Using Via-First Processes}

One of the first examples of via-first heterogeneous integration of MEMS with functional CMOS ICs is the monocrystalline silicon mirror array shown in Fig. 7. The array consists of $32 \times 32$ mirrors with dimensions of each mirror pixel of $1 \mathrm{~mm} \times 1 \mathrm{~mm}$ [6]. A variation of the via-first heterogeneous integration platform shown in Fig. 3 was used for the mirror integration [6]. In this process, mirrors, hinges and via bumps are partly pre-manufactured in a silicon wafer stack before the wafer stack is bonded to the CMOS IC wafer. Thereafter the bonded mirror wafer is thinned and further processed to form the mirror plates.

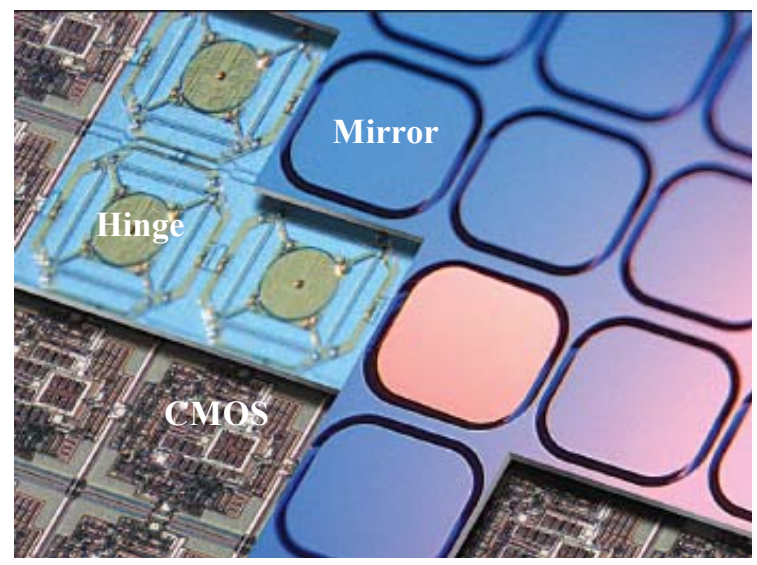

Figure 7. Mono-crystalline silicon mirror array integrated with CMOS ICs using a variation of the via-first heterogeneous integration platform depicted in Fig. 3. Each mirror is $1 \mathrm{~mm} \times 1 \mathrm{~mm}$ in size. [6]

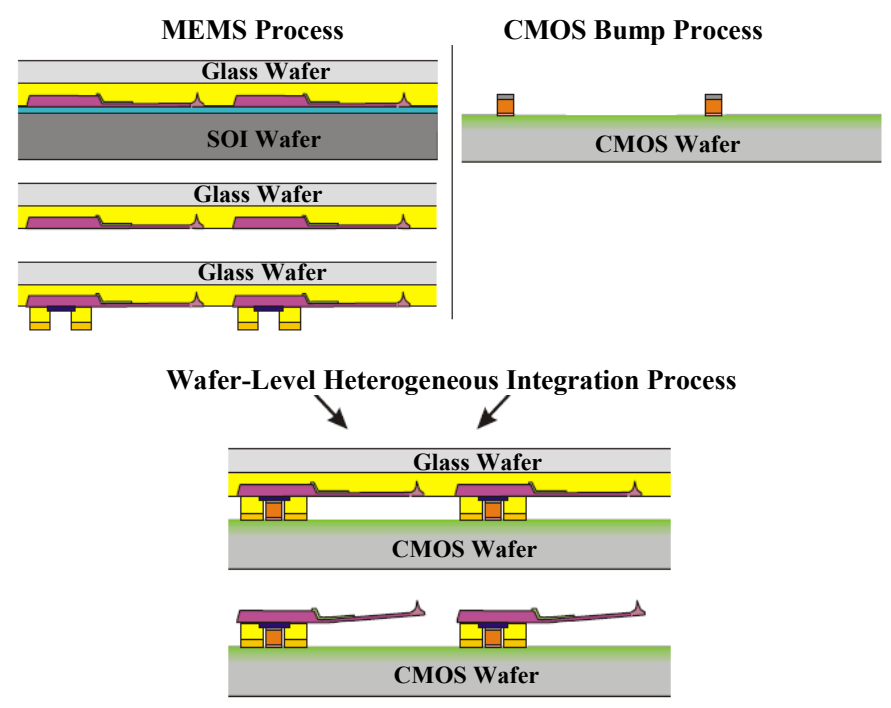

Figure 8. Wafer-level via-first heterogeneous integration platform for arrays of atomic force microscope tips. [7]

Another well-developed wafer-level via-first heterogeneous integration platform has been used for integrating arrays of atomic force microscope (AFM) tips made of mono-crystalline silicon on top of ICs [7] as shown in Fig. 8. The AFM tips are first fabricated on an SOI wafer and then transferred to a glass wafer by polyimide bonding and sacrificial etching of the SOI substrate. Thereafter metal studs and the patterned adhesive polyimide layer are formed on the backside of the AFM tips. Then the glass wafer with the AFM tips is aligned and bonded to the electronic IC wafer using combined solder bump bonding and polyimide adhesive bonding. Finally the transferred AFM tips are released from the glass wafer by laser de-bonding. The glass donor wafer is used to enable the laser de-bonding process and facilitate accurate wafer-to-wafer alignment before bonding. Fig. 9 shows SEM images of the integrated AFM tips. The dimensions of the vias of the AFM tips are $15 \mu \mathrm{m}$ in diameter, the AFM tip array has a pitch of $130 \mu \mathrm{m} \times 100 \mu \mathrm{m}$ and the mono-crystalline silicon AFM cantilevers are $300 \mathrm{~nm}$ thick.

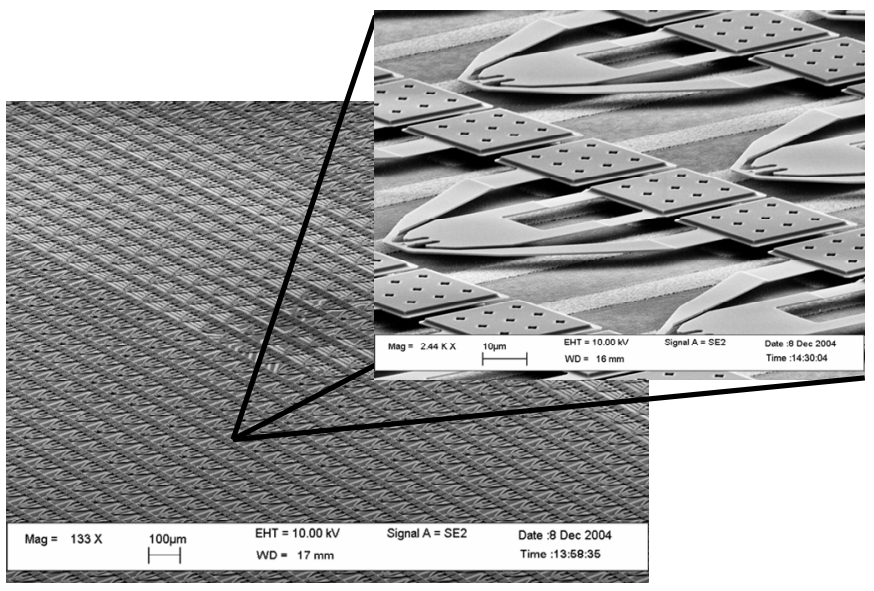

Figure 9. Arrays of atomic force microscope tips integrated on electronic ICs using the via-first heterogeneous integration platform shown in Fig. 8. [7]

\section{B. Wafer-Level Heterogeneous 3D Integration Platforms Using Via-Last Processes}

Demonstrations of via-first heterogeneous integration technologies for MEMS and NEMS include infrared bolometer arrays [8-14], arrays of tilting and piston-type micro-mirrors [14-18], micro-pirani vacuum gauges [19], RF MEMS [1, 20] and many more devices [1]. For most of these applications, adhesive wafer bonding has been used to join the MEMS and the IC wafers. Bonding with a polymer adhesive has the advantage that the MEMS or NEMS wafer and the CMOS IC wafer can be bonded with very high yield and without any surface pre-treatment or surface planarization [14, 21]. The first MEMS device that has been integrated with a fully functional CMOS IC using very large scale via-last heterogeneous $3 \mathrm{D}$ integration is the 1-megapixel monocrystalline silicon micro-mirror array shown in Fig. 11 [16]. Fig. 10 shows a detailed process flow for the integration of the mono-crystalline silicon micro-mirror array. The array has a pixel pitch of $16 \mu \mathrm{m} \times 16 \mu \mathrm{m}$, the silicon mirror membranes are $340 \mathrm{~nm}$ thick and have an extremely well defined distance of $700 \mathrm{~nm}$ to the addressing electrodes on the underlying CMOS ICs. The mirror vias have a diameter of $2 \mu \mathrm{m}$ and the torsional mirror hinges are $600 \mathrm{~nm}$ wide. 
Other examples of devices that have been demonstrated using via-first heterogeneous 3D integration technologies are hidden hinge, piston-type mirror array consisting of 96x96 mirrors with a pitch of $40 \mu \mathrm{m} \times 40 \mu \mathrm{m}$ as shown in Fig. 12a [18], and 320x240 pixels $\mathrm{Si} / \mathrm{SiGe}$ quantum-well infrared bolometers arrays as shown in Fig12b [8, 12, 13].

(a)

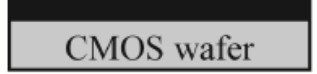

(b)

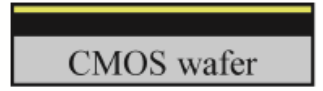

(c)

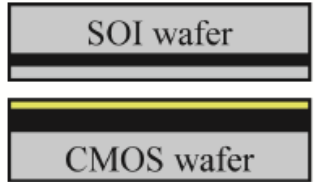

(d)

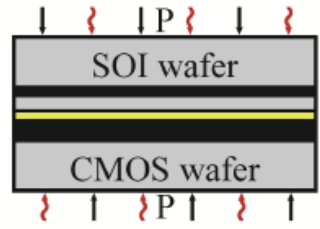

(e)

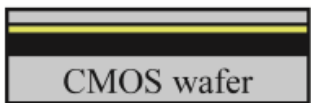

(f)

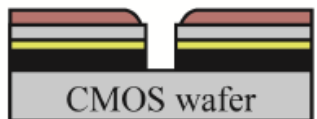

(g)

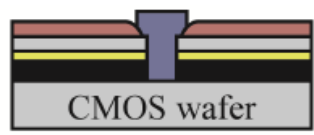

(h)

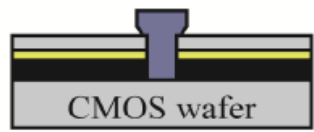

(i)

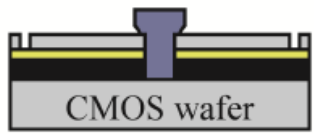

$\mathrm{SiO}_{2}$

Adhesive layer (BCB)

Metal

Silicon

Photoresist

(j)

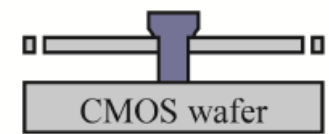

Figure 10. Simplified design flow. $\mathrm{SiO}_{2}$ deposition as sacrificial spacer material on the CMOS wafer (a), spin-coating of BCB as adhesive layer (b), adhesive bonding (c-d), etching of excess silicon and stripping of buried oxide layer (e), etching of via openings down to the CMOS electronics and broadening of photoresist-openings (f), metal deposition for via-filling with subsequent removal of excess metal (g), stripping of the photoresist layers (h), etching the mirror pattern into the silicon (i), final release etch to remove the sacrificial and adhesive layers (j). [16]

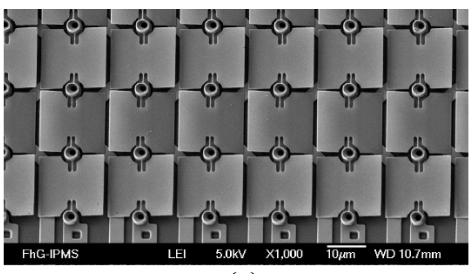

(a)

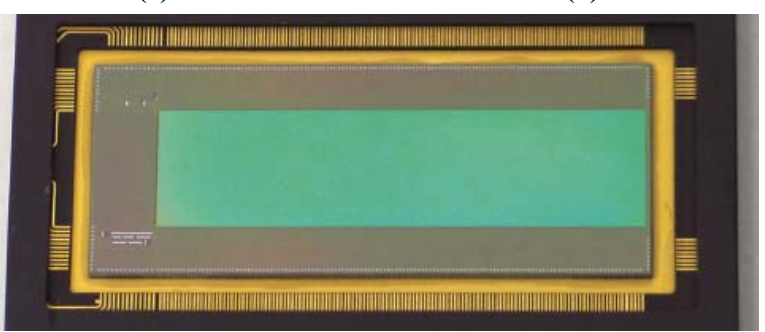

(c)

Figure 11. (a) 1-Mpixel tilting mono-crystalline silicon mirror array on CMOS driving electronics. (b) Focused ion beam etched cross-section of the CMOS substrate. (c) Package micro-mirror chip. [16]

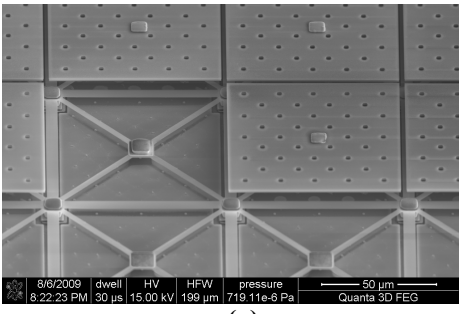

(a)

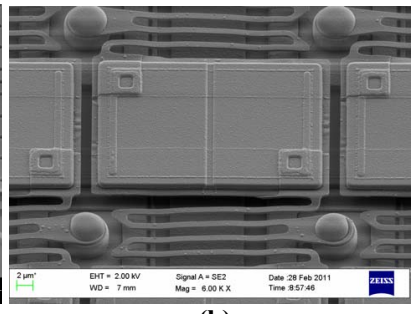

(b)
Figure 12. (a) Two-layer (hiden hinge) piston-type mono-crystalline silicon mirror array [18] and (b) IR SiGe quantum well bolometer array [13].

\section{Wafer-Level Heterogeneous 3D Integration Platforms Using Device-Distribution Approaches}

Conventional wafer-to-wafer heterogeneous integration platforms are an attractive and cost-efficient integration approach if the components manufactured on both wafers have high manufacturing yield and if they are comparable in size. However, in applications where the die size difference is large, wafer-level integration has a large cost penalty that makes it difficult to compete against the better established pick-andplace technology or, if the process allows it, monolithic integration. Indeed, a key prerequisite for wafer-level integration is that the chip or device to be transferred and the corresponding receiver die must have the same pitch on their respective wafers. This is cost effective in case of similar die size on the respective wafers as in the examples in sections A and B. However, when transferring devices that are much smaller than the receiving die, a lot of unused space is lost on the wafer carrying the devices to be transferred, resulting in cost inefficiency.

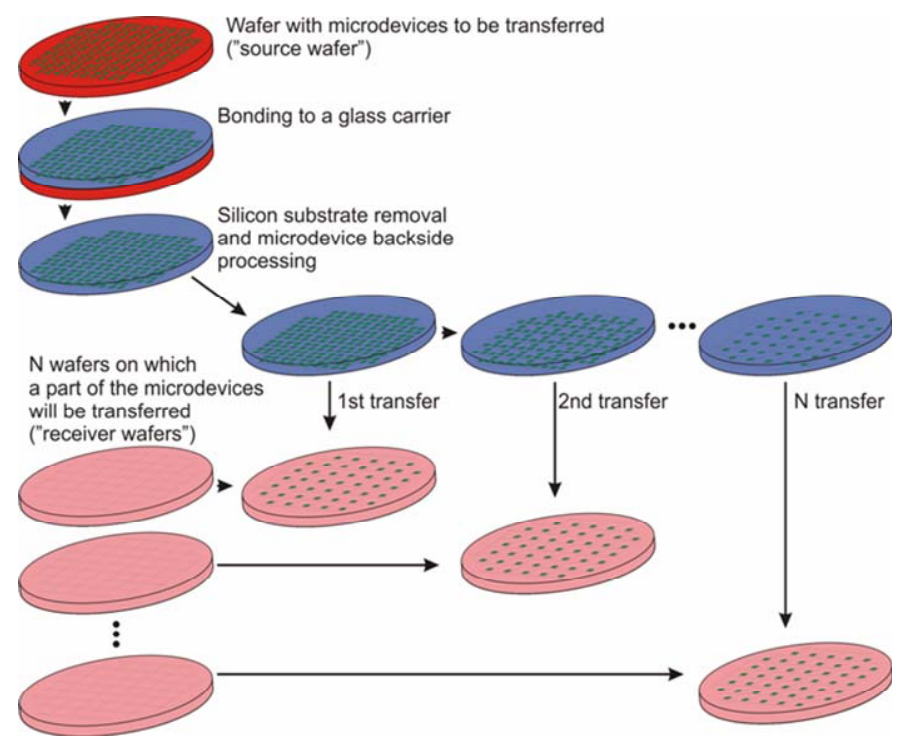

Figure 13. Wafer-to-wafer selective component distribution (microdevice distribution) from one donor wafer to several target wafers using a modified version of the process shown in Fig. 7. [22]

To address these shortcomings of wafer-level heterogeneous integration platforms, modified heterogeneous integration schemes have been proposed. These technologies 
include wafer-to-wafer selective component distribution, also called microdevice distribution (MD) technology [22-25], chip-to-wafer pick-and-place technologies [26, 27], the use of expandable handle substrates [28] and chip-to-wafer selfassembly techniques [1]. Fig. 13 and 14 shows a technology that is based on a selective transfer of devices to create the distribution function of a fraction of costly devices from one "source" wafer, having a high device density, to populate many receiver wafers having a lower device density. Hence the cost of the transferred device is distributed over the number of wafers populated. Fig. 14 shows how this method has been applied for the distribution of RF circuitry and RF MEMS switches [23]. The piezoelectrically actuated beams that are transferred to the RF substrates consist of a $1.5 \mu \mathrm{m}$ silicon nitride structural layer and a deposited layer of PZT, $\mathrm{Pb}\left(\mathrm{Zr}_{30} \mathrm{Ti}_{70}\right) \mathrm{O}_{3}$, which is grown onto a non-planar mixed interlayer of $\mathrm{TiO}_{2}$ and Ti/Pt. Distribution of AFM cantilever and SMA foils has also been demonstrated [24, 25].

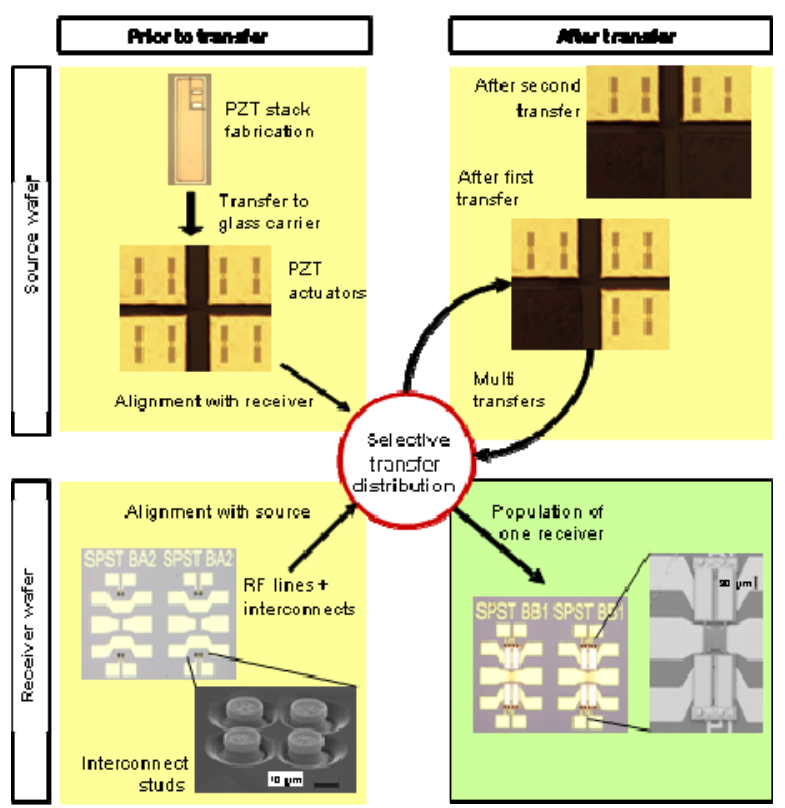

Figure 14. Wafer level distribution and interconnect of RF MEMS switches. Piezoelectrically actuated beams are fabricated on the source wafer and are selectively transferred to the receiver wafers. [22-25]

An elegant solution for via-last heterogeneous integration of expensive III-V materials that are only available in small wafer sized onto standard sized silicon wafers is shown in Fig. 15 [26]. In this approach, non-patterned dies (parts of a wafer) that contain the photonic layers of interest are placed on a polymer coated silicon target wafer in the areas were the components are needed. This can be done by inexpensive high-speed pick-and-place processes since only very low placement accuracies are required. After the dies are bonded, they are thinned and subsequently patterned and etched using wafer-level processes to form the photonic devices. A variation of this approach in which stretchable temporary transfer substrates are used is shown in Fig. 16 [28].
In addition to the presented integration processes, a number of interesting transfer printing and stamping transfer methods using via-last and via-first processes have been proposed to integrate such materials as carbon nanotubes and graphene sheets into silicon substrates [1, 29-31].

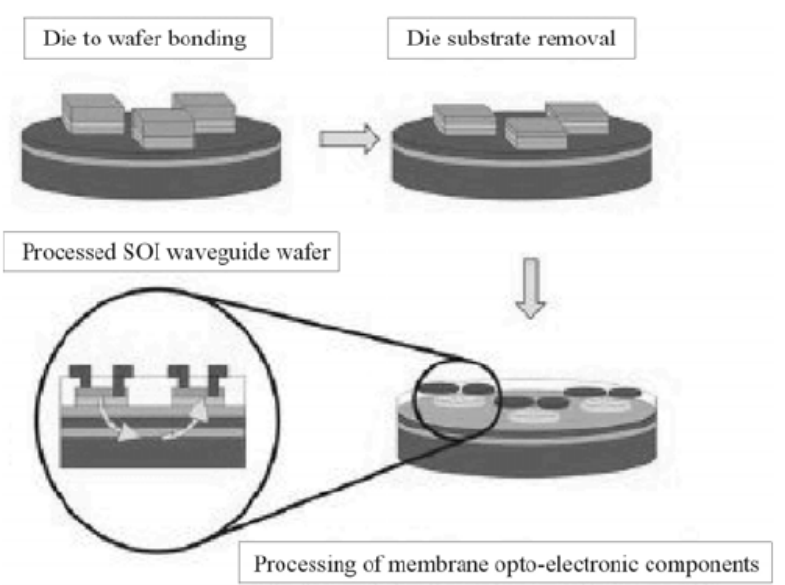

Figure 15. Die-to-wafer placement of component materials and subsequent wafer-level bonding, processing and interconnecting the integrated components. [26]

a)

b)

c)
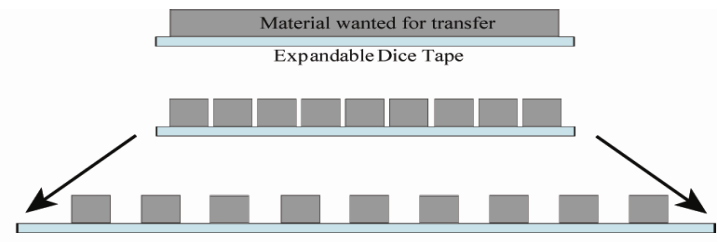

d)

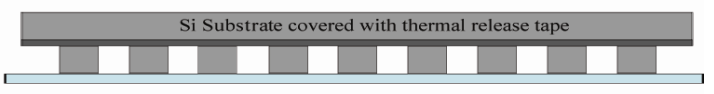

e)

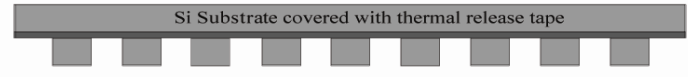

f)

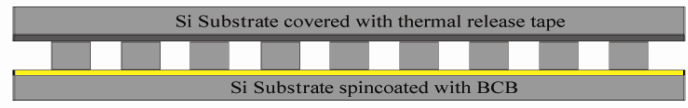

g)

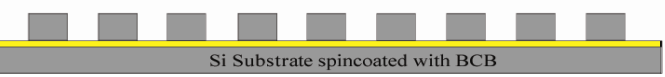

Figure 16. Process sequence of the transfer method based on stretchable temporary substrates. a) Source wafer on expandable dicing tape b) Dicing of wafer into dies c) Expansion of the tape to increase distance between dies d) Dies transferred to a temporary wafer covered by thermal release tape e) Removal of dicing tape f) Transfer of dies to a larger target wafer using adhesive wafer bonding g) Removal of the temporary wafer through thermal release . [28]

\section{DISCUSSION AND CONCLUSIONS}

Wafer-level heterogeneous integration technologies allow the combination of different high-performance materials and sub-systems to form advanced MEMS and NEMS devices. Thus, no compromise in the material selection has to be made and the same chip area can be used very efficiently both for the MEMS or NEMS parts and for the electronic parts for the 
signal read-out. These features enable complex, heterogeneous MEMS and NEMS solutions with high integration densities and high performances. For example, the close proximity of the sensor electrodes and the read-out electronics minimizes parasitic capacitances for capacitive MEMS and NEMS sensors, thus enabling sensing electrodes to be significantly reduced in size while maintaining sensitivity. Thus, the overall device dimensions and consequently their cost can be reduced.

It is remarkable that heterogeneously integrated MEMS devices have recently emerged and succeeded in very competitive markets such as for very high-volume consumer applications [2-4]. Some of the contributing factors to this commercial success of heterogeneous integration technologies are their compatibility with fables MEMS business models, the ability to utilize standard CMOS ICs from various sources and the possibility to shrink the overall MEMS or NEMS device dimensions and thus, enabling the combination of multiple sensors on a highly integrated single chip. In addition, an infrastructure with several MEMS foundries have emerged in recent years which has made fabless MEMS business models viable.

\section{ACKNOWLEDGMENT}

The authors thank their colleagues at the Microsystem Technology Lab at KTH Royal Institute of Technology in Stockholm, Sweden and at IBM Zurich Research Lab for many fruitful discussions. The information in this document is provided 'as is' and no guarantee or warranty is given that the information is fit for any particular purpose. The user thereof uses the information at its sole risk and liability.

\section{REFERENCES}

[1] M. Lapisa, G. Stemme F. Niklaus, "Wafer-Level Heterogeneous Integration for MOEMS, MEMS, and NEMS", IEEE Journal of Selected Topics in Quantum Electronics, Vol.17, No.3, pp.629-644, 2011.

[2] S. Nasiri, "New innovations in MEMS fabrications are responsible for meeting the demand for low-cost inertial sensors for consumer markets", White Paper, www.invensense.com.

[3] MEMS Process Review Report, "InvenSense IDG-300 Dual-Axis Angular Rate Gyroscope Sensor", MPR-0702-801, Chipworks, 2007.

[4] C.M. Liu, et al., "MEMS technology development and manufacturing in a CMOS foundry", IEEE Proc. Transducres 2011, pp.807-810, Beijing, China.

[5] F. Niklaus, et al., "A Method to Maintain Wafer Alignment Precision During Adhesive Wafer Bonding", Sensors \& Actuators A, Vol.107, No.3, pp.273-278, 2003

[6] J. Bryzek, et al., "Very Large Scale Integration of MOEMS Mirrors, MEMS Angular Amplifiers and High-Voltage, High-Density IC Electronics for Photonic Switching", Nanotech, Vol.2, pp.428-431, 2003.

[7] M. Despont, et al., "Wafer-Scale Microdevice Transfer/ Interconnect: its Application in an AFM-Based Data-Storage System", Journal of Microelectromechanical Systems, Vol.13, No.6, pp.895- 901, 2004.

[8] F. Niklaus, et al., "Characterization of transfer-bonded silicon bolometer arrays", Proc. SPIE 2004, Vol.5406, pp.521-530, Orlando, USA.

[9] F. Niklaus, et al., "Performance Model for Uncooled Infrared Bolometer Arrays and Performance Predictions of Bolometers Operating at Atmospheric Pressure", Infrared Physics and Technology, Vol.51, No.3, pp.168-177, 2008.

[10] C. Vieider, et al., "Low-Cost Far Infrared Bolometer Camera for Automotive Use", Proc. SPIE 2007, Vol.6542, pp.1L.1-1L.10, Orlando, USA.
[11] F. Niklaus, et al., "Uncooled Infrared Bolometer Arrays Operating in a Low to Medium Vacuum Atmosphere: Performance Model and Tradeoffs", Proc. SPIE 2007, Vol.6542, pp.1M.1-1M.12, Orlando, USA.

[12] F. Niklaus, C. Vieider, H. Jakobsen, "MEMS-Based Uncooled Infrared Bolometer Arrays - A Review", Proc. SPIE 2007, Vol. 6836, pp.0D10D15, Beijing, China.

[13] P. Ericsson, et al., "Toward $17 \mu \mathrm{m}$ Pitch Heterogeneously Integrated $\mathrm{Si} / \mathrm{SiGe}$ Quantum Well Bolometer Focal Plane Arrays", Proc. SPIE 2011, Vol.8012, Orlando, USA.

[14] F. Niklaus, et al., "Wafer Bonding with Nano-Imprint Resists as Sacrificial Adhesive for Fabrication of Silicon-On-Integrated-Circuit (SOIC) Wafers in 3D Integration of MEMS and ICs", Sensors and Actuators A: Physical, Vol.154, pp.180-186, 2009.

[15] F. Niklaus, S. Haasl, G. Stemme, "Arrays of Monocrystalline Silicon Micromirrors Fabricated Using CMOS Compatible Transfer Bonding", IEEE Journal of Microelectromechanical Systems, Vol.12, No.4, pp.465-469, 2003.

[16] F. Zimmer, et al., "One-Megapixel Monocrystalline-Silicon Micromirror Array on CMOS Driving Electronics Manufactured With Very LargeScale Heterogeneous Integration", IEEE Journal of Microelectromechanical Systems, Vol.20, No.3, pp.564-572, 2011.

[17] M.A. Lapisa, et al., "Drift-Free Micromirror Arrays Made of Monocrystalline Silicon for Adaptive Optics Applications", IEEE Journal of Microelectromechanical Systems, in print, 2012.

[18] M.A. Lapisa, et al., "Hidden-Hinge Micro-Mirror Arrays Made by Heterogeneous Integration of Two Mono-Crystalline Silicon Layers", Proc. IEEE MEMS 2011, pp.696-699, Cancun, Mexico.

[19] G. Schelcher, et al., "Modeling and characterization of micropirani vacuum gauges manufactured by a low-temperature film transfer process", Journal of Microelectromechanical Systems, Vol20, No.5, pp.1184-1191, 2011.

[20] T. Matsumura, et al., "Multi-Band Radio-Frequency Filters Fabricated Using Polyimide-Based Membrane Transfer Bonding Technology", Journal of Micromechanics and Microengineering, Vol.20, No.9, 095027, 2010.

[21] F. Niklaus, et al., "Adhesive wafer bonding", Journal of Applied Physics, Vol.99, No.1, pp.031101.1-031101.28, 2006.

[22] R. Guerre, et al., "Low-cost AFM cantilever manufacturing technology", Journal of Micromechanics and Microengineering, Vol.18, pp.115013, 2008.

[23] R. Guerre et al., "Wafer-level transfer technologies for PZT-based RF MEMS switches", Journal of Microelectromechanical Systems, Vol.19, No.3, pp.548-560, 2010.

[24] R. Guerre et al., "Selective transfer technology for microdevice distribution", Journal of Microelectromechanical Systems, Vol.17, No.1, pp.157-165, 2008.

[25] T. Grund et al., "Transfer bonding technology for batch fabrication of SMA microactuators", The European Physical Journal - Special Topics, Vol.158, No.1, pp.237-242, 2008.

[26] D. Liang, et al., "Hybrid Integrated Platforms for Silicon Photonics", Materials, Vol.3, pp.1782-1802, 2010.

[27] L. Jian, H. Takagi, R.Maeda, "Chip to wafer temporary bonding with self-alignment by patterned FDTS layer for size-free MEMS integration", IEEE Proc. Sensors 2011, pp.1121-1124, Limerick, Ireland.

[28] F. Forsberg, et al., "Heterogeneous Integration Technology for Combination of Different Wafer Sizes Using an Expandable Handle Substrate", Proc. IEEE MEMS 2011, pp.268-271, Cancun, Mexico.

[29] A. Javey, et al., "Layer-by-layer assembly of nanowires for threedimensional, multifunctional electronics", Nano Letters, Vol.7, No.3, pp.773-777, 2007.

[30] J. Yoon, et al., "GaAs photovoltaics and optoelectronics using releasable multilayer epitaxial assemblies", Nature, Vol.465, pp.329-333, 2010.

[31] X.L. Liang, et al., "Toward Clean and Crackless Transfer of Graphene”, ACS Nano, Vol.5, No.11, pp.9144-9153, 2011. 